Cancer of Unknown Primary (CUP): a Cancer Registry study of factors affecting access to diagnosis

S. Michael Crawford ${ }^{1}$ (corresponding author)

Jane Skinner ${ }^{2}$

Emma Coombes ${ }^{2}$

Andrew $\mathrm{P}$ Jones ${ }^{2}$

${ }^{1}$ Airedale General Hospital, Skipton Road, Steeton, Keighley, West Yorkshire, BD20 6TD

S.M.Crawford@doctors.org.uk

${ }^{2}$ Norwich Medical School, University of East Anglia, NR4 7TJ

Conflicts of Interest - none. 


\title{
Cancer of Unknown Primary (CUP): a Cancer Registry study of factors affecting access to
} diagnosis

S. Michael Crawford, Jane Skinner, Emma Coombes, Andrew P Jones

\begin{abstract}
A potential impact of the centralisation of cancer services in the UK is difficulty in gaining access for members of the population living far from them. This could lead to delayed presentation of cancer with more advanced disease and clinical deterioration at diagnosis. A patient may be recorded in the cancer registry as having cancer of unknown primary (CUP) if the clinical state at presentation precludes investigation. Other patients may be so recorded if investigation identifies sites of metastatic tumour but the primary is not found. We hypothesised that the first group would include more patients who experienced difficulties in gaining access to health services through residing in deprived areas or through poorer geographical access to healthcare facilities. To test this, we compared the diagnosis of CUP with a comparator tumour, carcinoma of the rectum where diagnosis is facilitated by an alarm symptom and where variations in access are lower. Records from the Northern and Yorkshire Cancer Registry from 1994-2002 with ICD 10 C77-C80 (CUP, including categories where investigations may have been incomplete or no primary cancer was found) and C20 (malignant neoplasm of rectum) were combined with travel time to services (primary care, secondary and tertiary services) and the Index of Multiple Deprivation (IMD). Logistic regression modelled predictors of CUP compared to C20 and, within CUP, the odds of a histological basis of diagnosis.
\end{abstract}

The registry classified 7,428 patients as $\mathrm{C} 80,8,849$ as $\mathrm{C} 77-79$, and 10,804 as $\mathrm{C} 20$. Compared to C20, the number of cases of C80 showed a statistically significant increasing trend with 
increasing travel time to primary care. Risk also increased strongly with age, and deprivation. Results for $\mathrm{C77-C79}$ were similar to those for $\mathrm{C} 80$, except that the travel time to primary care showed no effect. Considering all CUP alone, histological diagnosis significantly declined with travel time to the nearest hospital. There was no association with sex and the likelihood of histological diagnosis, but a marked decline with age, a downward trend with deprivation, and an increase when the nearest hospital was a cancer centre. These findings facilitate the understanding of factors associated with the group of patients that includes those with the least effective access to cancer services.

\section{Keywords}

Cancer of Unknown Primary, Cancer diagnosis, Rectal Cancer, Socioeconomic Deprivation, Access to Health Services, Primary Care. 


\section{Introduction}

Policy for the management of cancer in the UK has been driven by the observation that at the end of the previous century, survival rates from cancer were worse in the UK than in comparable European countries [1]. The reasons continue to be debated but, following the NHS Cancer Plan [2] policies sought to improve the quality of care by increasing specialist recruitment and enhancing services in selected centres. Some specialist diagnostic and therapeutic services for cancer have been concentrated in selected large hospitals, known as Designated Cancer Centres. Typical district general hospitals, known as Designated Cancer Units, focus on the management of common cancers where high volumes can be sustained.

A possible disadvantage of centralising services in this manner is that populations living further from specialist centres may have difficulties, including gaining transport to provide access to them for treatment, and such difficulties may consequently be associated with poorer disease prognosis $[3,4]$. Rural GPs have complained of the problems of gaining access to treatment for patients living in remoter areas far from cancer treatment centres [5] and a report from the Commission for Rural Communities has illustrated some adverse experiences of cancer sufferers and their carers living in rural England, with some patients facing round trips of 100 miles and the topography and quality of local roads further lengthening journey times [6]. There is accumulating evidence that longer travel times have negative effects on access to treatment and the outcome of care for patients with diagnosed cancer [7].

Campbell et al. [4] examined the relationship between survival and distance to cancer centres amongst 64,000 patients diagnosed with common cancers in Scotland between 1991 and 1995 . They found that increasing distance from a cancer centre was associated with less chance of diagnosis before death for stomach, breast, and colorectal cancers and poorer survival after 
diagnosis for prostate and lung cancers. A study of 5,147 cases of colorectal cancer in southern England [8] also found post-operative survival declined with increasing distance from a treatment centre.

Our previous study of cancers of the breast, bowel, lung, prostate and ovary, which used records from the former Northern and Yorkshire Cancer Registry and Information Service (NYCRIS), added to the evidence. We found that survival from cancers of the prostate was adversely associated with travel time to the patient's GP, as was the likelihood of presenting at late stage for breast or colorectal cancer [9]. Access to the centralised services of radiotherapy and thoracic surgery was shown to be reduced with increasing travel time, as was some chemotherapy [10] and the type of surgery used to treat breast cancer was influenced by access to radiotherapy [11]. These analyses adjusted for deprivation of the area of residence but more detailed analyses for lung cancer showed that the issues of access were minimal for the least deprived localities and greatest for the most deprived [12]. Similar observations were made for colonic but not rectal cancer; the symptom patterns of these tumours suggest that difficulty in suspecting the diagnosis was key [13]. There was also a tendency for those living furthest from a hospital to be recorded as having been diagnosed on the date of death [14].

Cancer service development since 2000 initially concentrated on hospital services but more recently the focus has shifted to the timing of diagnosis [15]. This requires improvement in the interface between primary care, where GPs have to decide if it is appropriate to consider the diagnosis in a patient, and secondary care, where the facilities for investigation are located. For patients with common epithelial cancers, those from deprived areas have been shown to be more likely to have their first hospital admission as an emergency event [16]. Current understanding of the role of primary care in the process is discussed in the Lancet Oncology 
Commission on the subject [17]. Practitioners in this discipline have a role throughout the cancer patient's journey but it is especially important leading up to the diagnosis.

There is concern about patients being admitted to hospital with previously undiagnosed cancer. The National Institute for Health and Care Excellence has issued a guideline for management of patients with malignancy of unknown primary [18], a decade after the Cancer Plan [2]. That document addresses the fact that this group of patients comprises two distinct entities; those patients whose primary site has not been identified because of presentation with very advanced disease and those in whom a primary cannot be identified. The first entity suggests that timely access to the diagnostic services of the Health Service has not been attained and in this study we investigate that process. We therefore decided to approach this entity in the same way and for the same time period over which we had looked at data concerning common cancer sites as an addition to the previous work. Taken as a whole, our body of work provides a detailed picture of the state of services at an important time point.

We hypothesised that cancers of unknown primary would include more patients who faced difficulties in gaining access to health services through residing in deprived areas or through having to travel further to cancer facilities. To test this we compared the diagnosis of Cancer of Unknown Primary (CUP) with a comparator tumour, carcinoma of the rectum, where associations with access are not strong [13]. Indeed, few patients with rectal cancer require multiple consultations before a diagnosis is reached [19], most likely because it has a signature symptom of rectal bleeding. Undiagnosed rectal cancer is therefore unlikely to contribute greatly to the total of CUP patients. The data we have analysed are from the time the Cancer Plan was being introduced and our results therefore form a historically relevant baseline against which the effects of that plan can be measured. 


\section{Materials and Methods}

\section{Setting}

The study drew patients from the area covered by the former Northern and Yorkshire Cancer Registry \& Information Service NYCRIS, which extended from the Scottish border to the Humber Estuary. The population covered was around 6.7 million. Approximately 17,500 new malignant cancer patients were assessed annually within this region during the period of study, and five year survival figures were lower than the national average for the majority of sites [20]. As well as widely distributed primary care services operated by general practitioners (GPs), the region contains 32 main acute hospitals (secondary care units) providing diagnostic and basic therapeutic services. Cancer Centres (specialist tertiary care units) are located in Hull, Leeds, Middlesbrough and Newcastle. The study area is predominantly rural and, in the counties of Northumberland and Cumbria, contains some of the most remote parts of Great Britain. The populations of some districts in those counties live an average distance of over $35 \mathrm{~km}$ from their nearest main acute hospital, compared to a national average distance of under $9 \mathrm{~km} \mathrm{[2].} \mathrm{The}$ area also includes the urban conurbations of Leeds and Tyneside and other industrial cities, with significant pockets of inner-city deprivation. Among the hospitals that are not cancer centres we have not separately analysed data concerning those that had an in-house oncology service.

These include several in West Yorkshire and Carlisle Infirmary, which has a radiotherapy facility.

\section{Subjects}

Records were supplied by NYCRIS for cancers registered from 1994 to 2002 . This period was chosen so that the findings were comparable with our previous work looking at different stages of the treatment/diagnosis pathway, described earlier [9-14]. NYCRIS supplied anonymous 
records of patients registered with the following ICD 10 classifications: C77 (Secondary and unspecified malignant neoplasm of lymph nodes), C78 (Secondary malignant neoplasm of respiratory and digestive organs), C79 (Secondary malignant neoplasm of other sites), and C80 (Malignant neoplasm without specification of site). C80 has the highest proportion of death certificate only and zero survival registrations and is likely to contain the patients whose presentation was with a clinical state that precluded further investigation. Data from ICD C20 Carcinoma of rectum were supplied and used as a comparator site.

The study was approved by the South Yorkshire Research Ethics Committee of the National Research Ethics Service.

\section{Accessibility and deprivation measures}

The travel time from the patients' home postcode to their GP and the nearest cancer centre and hospital was calculated using the ArcGIS v9.3 Geographical Information System (GIS) package (ESRI Inc). In order to do this, a digital representation of the road network was constructed using the Ordnance Survey Meridian dataset [21] and network routing algorithms were used in the GIS to identify the most direct route along the road network from each patient's home to their GP, the nearest cancer centre and nearest hospital. The total travel time in minutes for that route was then computed based on route length and the mix of road types. All calculations assumed car travel.

As a measure of neighbourhood material deprivation, an Index of Multiple Deprivation (IMD) score [22] was calculated for each individual based on the Census Lower Super Output Area zone that their postcode fell within. We computed scores for the index minus the access to 
services domain contribution to avoid duplication with our own access measures. To preserve anonymity, postcodes were removed from the records before they left the Registry for statistical analysis.

\section{Analysis}

Logistic regression was used to model predictors of CUP compared to carcinoma of the rectum. Models for C77-79 and C80 separately were adjusted for sex, age group, material deprivation quartile, and travel time to GP quartile. For CUP patients only, we also modelled the travel time to the nearest hospital and whether the nearest hospital was a cancer centre. Both of these models were adjusted for sex, age group and deprivation quartile. Tests for trend were carried out by fitting the values of the quartile number as a continuous variable. All analyses were carried out in Stata (version 14).

\section{Results}

In the dataset studied, 7,428 patients were classified as $C 80,8,849$ as $C 77-79$, and 10,804 as C20. Characteristics of patients in the analyses are summarised in Table 1. C20 patients were disproportionately male (62.2\%). CUP patients tended to be older, especially those classified as C80. The table shows that fewer of the participants had travel time to their GP available than had the other travel time measurements as the postcode of the GP that these patients attended was not recorded in the cancer registry records.

Table 2 gives the results from the logistic regression model, with the odds ratios for $\mathrm{C} 80$ compared to C20 for sex, age group, deprivation quartile and travel time to GP quartile. Travel time to GP showed a shallow gradient; odds ratios (ORs) $(1.00,1.09,1.14)$ for quartiles $2-4$ 
respectively, compared to the closest quartile 1 , with $p$ (trend) $=0.007$. Compared to $C 20$, there was a significant protective effect against being classified as C80 in males, but this is partly an artefact of the high number of males in the C20 group which reflects the known sex ratio for rectal cancer. Risk of classification as C80 compared to C20 increased strongly with age, with the test for trend being highly significant $(<0.001)$. Risk of diagnosis of $\mathrm{C} 80$ compared to $\mathrm{C} 20$ also increased with deprivation, $\mathrm{p}($ trend $)<0.001$.

Table 3 shows the corresponding results for C77-C79. These were similar to those for C80, except that the travel time to GP showed no association, $p($ trend) $=0.241$.

Table 4 shows the results of a logistic regression considering all CUP patients alone, and modelling the chance of a histological basis of diagnosis. There was no association with sex and the likelihood of histological diagnosis, but an extremely strong association with age, with an OR as low as 0.063 for the $80+$ group and $p($ trend $)<0.001$. Histological diagnosis declined with deprivation, $\mathrm{p}$ (trend) $<0.001$ and less markedly with travel time to the nearest hospital; ORs $(0.96,0.88,0.87)$ for quartiles $2-4, p($ trend $)=0.001$. There was a small but significant increased chance of a histological diagnosis if the nearest hospital was a cancer centre, OR=1.096 (95\% Cl 1.012-1.087).

The fitting of interaction terms suggested that deprivation did not moderate the association with travel time in any of the models.

\section{Discussion}

Main findings of the study 
Compared to rectal cancer, risk of CUP diagnosis increased with age, deprivation and, for C80, travel time to GP. For CUP patients, the chance of a histological basis of diagnosis decreased with age, deprivation and travel time to hospital and whether or not the nearest hospital was a cancer centre.

\section{What is already known on this topic?}

An American study found that a higher proportion of CUP was diagnosed in the elderly, females, black people and residents of less affluent or less educated counties [23]. A recent study of Scottish Cancer Registry data from 1961-2010 found that during 2001-2010, age-standardised rates of CUP were higher in the most compared with the least deprived quintile of the population [24]. They also found that CUP was unusual in people younger than 40 years and that rates increased quite steeply with age, although with some variation by sex and decade of incidence.

\section{What this study adds}

To our knowledge, no other studies have looked at travel time to locations of healthcare provision and CUP, or investigated the factors associated with the likelihood of a histological diagnosis in CUP patients. This is the last in a series of studies based on cancer registry data from NYCRIS covering the period at the turn of the $20^{\text {th }}$ century when the leadership of the NHS was recognising the shortfall in cancer survival that characterised the UK in comparison with other Western European economies. Whilst the Cancer Plan [2] was a major step in the process of improving hospital-based services concerned with cancer treatment, the question of what happens before the patient is seen in a hospital was not a focus until later that decade [15]. 
Even now, GPs are aware of their paramount role as gatekeepers of the NHS whilst acting appropriately to allow timely diagnosis of cancer [17].

Paramount in the development of understanding the importance of the timing of diagnosis was the recognition that much of the international difference in survival was accounted for by deaths in the first year. Møller et al showed that differences in survival of patients with bowel cancer within England between socioeconomic groups were also apparent in the short term, that is poorer people are more likely to experience early death and the difference in survival between socioeconomic groups is much less for those who survive the first year [25]. Early death is likely to be a consequence of very advanced disease at the time of diagnosis. Our previous work has shown that there are differences in access to treatment for bowel cancer associated with socioeconomic status but these are relatively small for rectal cancer [13]. This led us to use rectal cancer as the reference group in this study.

In studying patients registered as having cancer without a primary site being specified we acknowledge that when the diagnosis is not confirmed histologically, some patients will not actually have cancer; the diagnosis is inferred from clinical and radiological findings. Indeed, we have two groups of patients which overlap in the ICD classifications. Some patients are not fully investigated, commonly because of severe comorbidity or because the underlying cancer is causing them to be very ill. This category is expected to form the bulk of the C 80 classification. It represents patients in whom the cancer diagnostic services have, for whatever reason, been least successful.

The second group consists of patients in whom investigations fail to reveal the primary; this is a recognised entity requiring specialist treatment. These patients will mostly be included in categories C77-C79 along with some patients in C80. For these patients palliative systemic 
therapy is available but this should not be given without a histological diagnosis. Attainment of this step is therefore a measure of active management of the patient. In addition to the strong effects of age and deprivation, we have shown that whether or not the nearest hospital is a cancer centre influences it. This is likely to reflect the perceived utility of the precise diagnosis which in turn reflects what treatment is thought potentially appropriate. Whether or not an oncological opinion is brought is readily available will be relevant here. In the period since these patients were registered we have seen the development of multidisciplinary teams, in fulfilment of the Cancer Plan [2] and of acute oncology services. These should reduce the difference between the types of institutions.

It may be argued that new investigative techniques may decrease the number of patients designated CUP. The NICE Guidance proposes a conservative approach to investigation in the light of limited evidence [18]. It does not advocate the use of magnetic resonance imaging except in the assessment of lymphadenopathy that might be due to breast cancer. Similarly, recommendations for imaging that includes positron emission tomography advises its use only in cervical lymphadenopathy when an aerodigestive primary is possible. It is therefore not likely that their introduction to routine practice will have had an impact on the situation we have found.

The delivery of up-to-date cancer services has been a major endeavour within the National Health Service within the past 20 years after it became apparent that there were weaknesses compared with comparable European countries. Lyratzopoulos et al have explored the issue of diagnostic delay when patients have come to the attention of a GP [26]. They argue that diagnosis may be swifter if facilitated by decision support interventions, better interactions between generalists and specialists, and easier access to diagnostics. In this respect, the 
characteristics we have found to be associated with a higher likelihood of being diagnosed with CUP are very similar to the characteristics associated with early death after a diagnosis of lung cancer [27].

Much has been done to improve the process for patients who come to specialist attention and current efforts are aimed at improving the timeliness of the initiation of diagnostic processes. To this must be added the need to understand the barriers that prevent potential cancer patients from seeking an explanation for their symptoms. We have studied a group of patients which includes those who are least well served by the process. This sets a baseline against which improvements in access to diagnosis can be measured. In particular, we found a higher likelihood of histological confirmation of CUP, with the implication of greater diagnostic endeavour and expertise, when the nearest hospital was a cancer centre; yet this disparity may become less pronounced with enhanced performance throughout the NHS. Further, our findings suggest that the increased travel times typically experienced by patients attending cancer units may be detrimental to some. In addition, whilst the association between CUP and socioeconomic status has been noted before, with CUP generally being over-represented by those from more deprived backgrounds [28], we believe this is one of the first studies to assess socioeconomic status in connection with access to services.

\section{Limitations of this study}

We were not able to separate those patients presenting too unwell for investigation to be appropriate from those who were able to be investigated and were not, and those who were thoroughly investigated but no primary tumour was found. We would anticipate that C80 would mostly contain the first group and that most of the second and third groups would be in C77-79. 
For comparison with previous results, this study was based on data for patients diagnosed between 1994 and 2002. This has the advantage, however, of establishing a baseline to measure the effects of changes in policy and practice. In fact, the focus on diagnosis in UK policy and the formulation of a policy for Metastasis of Unknown Origin (MUO) occurred much more recently with guidance being published in 2010 [18] and so our observations are likely to have retained their relevance until the start of the current decade. Studies of current patients will assess what effect they are having. Our own recent work in lung cancer confirms that major geographical variations in access to services persisted at least until 2010 in spite of the efforts to develop services [29].

Our study is cross-sectional in nature, so we cannot determine if the associations we have seen are causal. We had no information on patient ethnicity or individual conditions or characteristics beyond age and sex, and we relied on an area-based measure of deprivation, the IMD. This was a surrogate for individual measures of disadvantage, which were unavailable. Our measure of deprivation may thus be subject to the ecological fallacy [30], where patients living in a deprived area were not themselves deprived, but our large sample size reduces the expected effects of this factor. We had data on travel time to GP for a smaller number of patients $(5697,64 \%$ of the total); those for whom this information was available may not be representative of all patients.

Much of the cancer registry information is obtained by individuals who are not clinicians and who may not have access to every detail of the patient's clinical record. This will result in some patients inappropriately being recorded as CUP. One Australian study [31] audited 574 cancer registry CUP diagnoses (C80.9) and found that $30.0 \%$ of cases were reclassified to a known primary site, mostly cutaneous, and $1.6 \%$ were found to be non-malignant; some of our patients 
will not have had cancer and some will have had diagnoses unknown to us. If the distribution of such error is random, the effect will have been to dilute the associations between age, travel time, deprivation and institution type that we have seen.

\section{Conclusions}

We have found that, in the time period we have studied and compared to rectal cancer, risk of CUP diagnosis increases for C80 with travel time to GP. For all registry categories of CUP patients, the chance of a histological basis of diagnosis decreases with travel time to hospital and increases when that hospital is a cancer centre. As the NHS continues to press for timelier ascertainment of cases of cancer in order that patients can best benefit from treatment it should be expected that the adverse trends that we have shown for deprivation and travel time to care will be reduced. It is also important to recognise that the gateway to care will be through the hospital first encountered, so all NHS institutions providing a diagnostic service should be able to do this effectively whether or not they are designated cancer centres.

\section{Acknowledgements}

We are grateful for the assistance of Ruth Burns and Sarah Lawton of NYCRIS in providing the database. No specific funding was received for this study. 
Table 1: Characteristics of study participants

\begin{tabular}{|c|c|c|c|c|c|c|}
\hline & \multicolumn{2}{|c|}{ C77-C79 } & \multicolumn{2}{|c|}{$\mathrm{C} 80$} & \multicolumn{2}{|c|}{$\mathrm{C} 20$} \\
\hline & $\mathrm{n}$ & $\%$ & $\mathrm{n}$ & $\%$ & $\mathrm{n}$ & $\%$ \\
\hline \multicolumn{7}{|l|}{ Sex } \\
\hline Female & 4,459 & 50.4 & 4,057 & 54.6 & 4,085 & 37.8 \\
\hline Male & 4,390 & 49.6 & 3,371 & 45.4 & 6,719 & 62.2 \\
\hline \multicolumn{7}{|l|}{ Age at diagnosis (y) } \\
\hline$<50$ & 395 & 4.5 & 269 & 3.6 & 591 & 5.5 \\
\hline $50-59$ & 919 & 10.4 & 590 & 7.9 & 1,432 & 13.3 \\
\hline $60-69$ & 1,989 & 22.5 & 1,310 & 17.6 & 2,890 & 26.8 \\
\hline $70-79$ & 3,215 & 36.3 & 2,647 & 35.6 & 3,626 & 33.6 \\
\hline $80+$ & 2,331 & 26.3 & 2,612 & 35.2 & 2,265 & 21.0 \\
\hline \multicolumn{7}{|l|}{ Deprivation (IMD Score) } \\
\hline Quartile 1 (least deprived) & 2,140 & 24.2 & 1,556 & 21.0 & 3,080 & 28.5 \\
\hline Quartile 2 & 2,227 & 25.2 & 1,882 & 25.3 & 2,655 & 24.6 \\
\hline Quartile 3 & 2,260 & 25.5 & 1,906 & 25.7 & 2,607 & 24.1 \\
\hline Quartile 4 (most deprived) & 2,222 & 25.1 & 2,084 & 28.1 & 2,462 & 22.8 \\
\hline \multicolumn{7}{|l|}{ Estimated travel time to GP } \\
\hline Quartile 1 (0-<2.9 mins) & 1,433 & 25.2 & 1,081 & 25.4 & 2,333 & 24.8 \\
\hline Quartile 2 (2.9-<5.3 mins) & 1,472 & 25.8 & 1,018 & 23.9 & 2,353 & 25.0 \\
\hline Quartile 3 (2.3-<9.1 mins) & 1,368 & 24.0 & 1,101 & 25.9 & 2,382 & 25.3 \\
\hline Quartile 4 (9.1-231.7 mins) & 1,424 & 25.0 & 1,056 & 24.8 & 2,357 & 25.0 \\
\hline \multicolumn{7}{|c|}{ Travel time to nearest cancer centre } \\
\hline Quartile 1 (0.1-<6.1 mins) & 2,209 & 25.0 & 1,970 & 26.5 & 2,649 & 24.5 \\
\hline Quartile 2 (6.1-<27.4 mins) & 2,260 & 25.5 & 1,909 & 25.7 & 2,640 & 24.4 \\
\hline Quartile 3 (27.4-<38.8 mins) & 2,156 & 24.4 & 1,795 & 24.2 & 2,737 & 25.3 \\
\hline Quartile 4 (38.8-195.0 mins) & 2,224 & 25.1 & 1,754 & 23.6 & 2,778 & 25.7 \\
\hline \multicolumn{7}{|l|}{ Travel time to nearest hospital } \\
\hline Quartile 1 (0.1-<7.6 mins) & 2,272 & 25.7 & 1,889 & 25.4 & 2,416 & 22.4 \\
\hline Quartile 2 (7.6-<11.6 mins) & 2,194 & 24.8 & 1,908 & 25.7 & 2,754 & 25.5 \\
\hline Quartile 3 (11.6-<17.1 mins) & 2,214 & 25.0 & 1,854 & 25.0 & 2,771 & 25.7 \\
\hline Quartile 4 (17.1-117.5 mins) & 2,169 & 24.5 & 1,777 & 23.9 & 2,863 & 26.5 \\
\hline \multicolumn{7}{|l|}{ Histological basis of diagnosis } \\
\hline No & 4,542 & 51.3 & 5,639 & 75.9 & & \\
\hline Yes & 4,307 & 48.7 & 1,789 & 24.1 & & \\
\hline
\end{tabular}


Table 2: Odds ratio for cancer of unknown primary (compared to C20, rectal carcinoma), adjusted for sex, age group, deprivation quartile and travel time to GP quartile for ICD code $\mathrm{C} 80(n=13,681)$

\begin{tabular}{lrrrr}
\hline & Odds ratio & $95 \% \mathrm{Cl}$ & P-value & P-trend \\
\hline Sex & & & & \\
$\quad$ Female & 1.000 & & & \\
$\quad$ Male & 0.561 & $0.520-0.605$ & $<0.001$ & \\
& & & & \\
Age at diagnosis (y) & & & & \\
$\quad<50$ & 1.000 & & 0.802 & \\
$50-59$ & 1.030 & $0.819-1.294$ & 0.063 & \\
$60-69$ & 1.219 & $0.990-1.503$ & & \\
70-79 & 1.841 & $1.504-2.253$ & $<0.001$ & \\
80+ & 2.455 & $2.000-3.014$ & $<0.001$ & $<0.001$ \\
& & & & \\
Deprivation (IMD Score) & & & & \\
$\quad$ Quartile 1 (least deprived) & 1.000 & & & \\
$\quad$ Quartile 2 & 1.532 & $1.371-1.712$ & $<0.001$ & \\
$\quad$ Quartile 3 & 1.739 & $1.557-1.941$ & $<0.001$ & \\
$\quad$ Quartile 4 (most deprived) & 2.067 & $1.853-2.305$ & $<0.001$ & $<0.001$ \\
& & & & \\
Travel time to GP & & & & \\
$\quad$ Quartile 1 (0-<2.9 mins) & 1.000 & & & \\
$\quad$ Quartile 2 (2.9-<5.3 mins) & 1.003 & $0.902-1.115$ & 0.959 & \\
Quartile 3 (2.3-<9.1 mins) & 1.091 & $0.983-1.212$ & 0.102 & \\
Quartile 4 (9.1-231.7 mins) & 1.135 & $1.020-1.262$ & 0.020 & 0.007 \\
\hline
\end{tabular}


Table 3: Odds ratio for cancer of unknown primary (compared to rectal carcinoma, C20), adjusted for sex, age group, deprivation quartile, travel time to GP quartile for ICD codes C77C79 $(n=15,122)$

\begin{tabular}{lrrrr}
\hline & Odds ratio & $95 \% \mathrm{Cl}$ & P-value & P-trend \\
\hline Sex & & & & \\
$\quad$ Female & 1.000 & & & \\
$\quad$ Male & 0.612 & $0.572-0.655$ & 0.000 & \\
& & & & \\
Age at diagnosis (y) & & & & \\
$\quad<50$ & 1.000 & & 0.769 & \\
$50-59$ & 1.029 & $0.852-1.241$ & 0.082 & \\
60-69 & 1.165 & $0.981-1.385$ & & \\
70-79 & 1.458 & $1.232-1.725$ & $<0.001$ & \\
$80+$ & 1.594 & $1.341-1.895$ & $<0.001$ & $<0.001$ \\
& & & & \\
Deprivation (IMD score) & & & & \\
$\quad$ Quartile 1 (least deprived) & 1.000 & & & \\
$\quad$ Quartile 2 & 1.342 & $1.219-1.477$ & $<0.001$ & \\
$\quad$ Quartile 3 & 1.415 & $1.285-1.557$ & $<0.001$ & \\
$\quad$ Quartile 4 (most deprived) & 1.598 & $1.453-1.758$ & $<0.001$ & $<0.001$ \\
& & & & \\
Travel time to GP & & & & \\
$\quad$ Quartile 1 (0-<2.9 mins) & 1.000 & & & \\
$\quad$ Quartile 2 (2.9-<5.3 mins) & 1.061 & $0.966-1.166$ & 0.214 & \\
$\quad$ Quartile 3 (2.3-<9.1 mins) & 0.992 & $0.902-1.091$ & 0.862 & \\
Quartile 4 (9.1-231.7 mins) & 1.087 & $0.988-1.196$ & 0.088 & 0.241 \\
\hline
\end{tabular}


Table 4: Odds ratio for histological basis of diagnosis adjusted for sex, age group, deprivation quartile, travel time to nearest hospital quartile and whether the nearest hospital was a cancer centre for ICD codes C77-C80 (n=16,277)

\begin{tabular}{|c|c|c|c|c|}
\hline & Odds ratio & $95 \% \mathrm{Cl}$ & P-value & P-trend \\
\hline \multicolumn{5}{|l|}{ Sex } \\
\hline Female & 1.000 & & & \\
\hline Male & 0.978 & $0.913-1.048$ & 0.543 & \\
\hline \multicolumn{5}{|l|}{ Age at diagnosis (y) } \\
\hline$<50$ & 1.000 & & & \\
\hline $50-59$ & 0.521 & $0.422-0.644$ & $<0.001$ & \\
\hline $60-69$ & 0.321 & $0.264-0.390$ & $<0.001$ & \\
\hline $70-79$ & 0.152 & $0.126-0.184$ & $<0.001$ & \\
\hline $80+$ & 0.063 & $0.052-0.076$ & $<0.001$ & $<0.001$ \\
\hline \multicolumn{5}{|l|}{ Deprivation (IMD score) } \\
\hline Quartile 1 (least deprived) & 1.000 & & & \\
\hline Quartile 2 & 0.752 & $0.681-0.830$ & $<0.001$ & \\
\hline Quartile 3 & 0.667 & $0.604-0.736$ & $<0.001$ & \\
\hline Quartile 4 (most deprived) & 0.618 & $0.559-0.684$ & $<0.001$ & $<0.001$ \\
\hline \multicolumn{5}{|l|}{ Travel time to nearest hospital } \\
\hline Quartile 1 (0.1-<7.6 mins) & 1.000 & & & \\
\hline Quartile $2(7.6-<11.6$ mins $)$ & 0.956 & $0.868-1.052$ & 0.358 & \\
\hline Quartile 3 (11.6-<17.1 mins) & 0.881 & $0.800-0.971$ & 0.011 & \\
\hline Quartile 4 (17.1-117.5 mins) & 0.876 & $0.792-0.968$ & 0.010 & 0.003 \\
\hline \multicolumn{5}{|c|}{ Nearest hospital was a cancer centre } \\
\hline No & 1.000 & & & \\
\hline Yes & 1.096 & $1.012-1.187$ & 0.025 & \\
\hline
\end{tabular}




\section{References}

[1] Sant M, Capocaccia R, Coleman MP, Berrino F, Gatta G, Micheli et al. Cancer survival increases in Europe, but international differences remain wide. Eur J Cancer 2001;37: 1659-67.

[2] Department of Health. The NHS cancer plan: a plan for investment, a plan for reform. London, HMSO. 2000

[3] Bain NS. Campbell NC. Treating patients with colorectal cancer in rural and urban areas: a qualitative study of the patients' perspective. Fam Pract 2000; 17: 475-9.

[4] Campbell NC, Elliott AM, Sharp L, Ritchie LD, Cassidy J, Little J.. Rural factors and survival from cancer: analysis of Scottish cancer registrations. Br J Cancer 2000;82: 1863-6.

[5] Baird A G, Donnelly CM, Miscampell NT, Wemyss HD, . Centralisation of cancer services in rural areas has disadvantages. BMJ 2000; 320: 717.

[6] Commission for Rural Communities. Insights from users and providers of cancer care in rural England: summary report. Cheltenham, Commission for Rural Communities. 2010

[7] Commission for Rural Communities. Service needs and delivery following cancer diagnosis: evidence based review. Cheltenham, Commission for Rural Communities, 2009.

[8] Kim YE, Gatrell AC, Francis BJ. The geography of survival after surgery for colo-rectal cancer in southern England. Soc Sci Med 2000; 50: 1099-107.

[9] Jones AP, Haynes R, Sauerzapf V, Crawford SM, Zhao H, Forman D. Travel time to hospital and treatment for breast, colon, rectum, lung, ovary and prostate cancer. Eur J Cancer 2008; 44: 992-9.

[10] Jones AP, Haynes R, Sauerzapf V, Crawford SM, Zhao H, Forman D. Travel times to health care and survival from cancers in Northern England. Eur J Cancer 2008; 44: 269-274.

[11] Sauerzapf VA, Jones AP, Haynes R, Crawford SM, Forman D. Travel time to radiotherapy and uptake of breast-conserving surgery for early stage cancer in Northern England. Health Place 2008; 14: 424-433.

[12] Crawford SM, Sauerzapf V, Haynes R, Zhao H, Forman D, Jones AP. Social and geographical factors affecting access to treatment of lung cancer. Br J Cancer 2009; 101: 897-901.

[13] Crawford SM, Sauerzapf V, Haynes R, Forman D, Jones AP. Social and geographical factors affecting access to treatment of colorectal cancer: a cancer registry study. BMJ Open 2012; 2: e000410.

[14] Jones AP, Haynes R, Sauerzapf V, Crawford SM, Forman D. Geographical access to healthcare in Northern England and post-mortem diagnosis of cancer. J Public Health (Oxf) 2010; 32: 532-537. 
[15] Richards, MA. The size of the prize for earlier diagnosis of cancer in England. Br J Cancer 2009; 101 Suppl 2: S125-129.

[16] Raine R, Wong W, Scholes S, Ashton C, Obichere A, Ambler G. Social variations in access to hospital care for patients with colorectal, breast, and lung cancer between 1999 and 2006: retrospective analysis of hospital episode statistics BMJ 2010; 340: b5479.

[17] Rubin G, Berendsen A, Crawford SM, et al. The expanding role of primary care in cancer control. The Lancet Oncology Commission. Lanc Oncol 2015; 16: 1231-72.

[18] National Institute for Health and Care Excellence. NICE clinical guideline 104: Metastatic malignant disease of unknown primary origin. London, NICE. 2010

[19] Lyratzopoulos G, Neal RD, Barbiere JM, Rubin GP, Abel GA.Variation in number of general practitioner consultations before hospital referral for cancer: findings from the 2010 National Cancer Patient Experience Survey in England. Lancet Oncol. 2012;13:353-65.

[20] Northern \& Yorkshire Cancer Registry \& Information Service. Cancer treatment policies and their effects on Survival: Colorectal. Leeds, NYCRIS. 2000

[21] Ordnance Survey (2016) Meridian Data. Data description available from https://www.ordnancesurvey.co.uk/business-and-overnment/products/meridian2.html Website accessed 27-01-2016.

[22] Office of the Deputy Prime Minister . The English indices of deprivation 2004 (revised). Wetherby, ODPM Publications.

[23] Urban D, Rao A, Bressel M, Lawrence YR, Mileshkin L. Cancer of unknown primary: a population-based analysis of temporal change and socioeconomic disparities. Br J Cancer 2013; 109: 1318-24.

[24] Brewster DH, Lang J, Bhatti LA, Thomson CS, Oien KA. Descriptive epidemiology of cancer of unknown primary site in Scotland, 1961-2010. Cancer Epidemiol 2014; 38: 227-34.

[25] Møller H, Sandin F, Robinson D, et al. Colorectal cancer survival in socioeconomic groups in England: variation is mainly in the short term after diagnosis. Eur J Cancer 2011;48:46-53.

[26] Lyratzopoulos G, Wardle J, Rubin G. Rethinking diagnostic delay in cancer: how difficult is the diagnosis? BMJ 2014;349:g475-82.

[27] O'Dowd EL, McKeever TM, Baldwin DR et al. What characteristics of primary care and patients are associated with early death in patients with lung cancer in the UK? Thorax 2015;70:161-168.

[28] Luke C, Koczwara B, Karapetis C, et al. Exploring the epidemiological characteristics of cancers of unknown primary site in an Australian population: implications for research and clinical care. Aust N Z J Public Health. 2008;32:383-9. 
[29] Murage P, Crawford M, Bachmann M, Jones AP. Geographical disparities in access to lung cancer services in England. British Thoracic Oncology Group Annual Conference Dublin 27-29 January 2016. Lung Cancer 91, 2016 (suppl 1) pS39.

[30] Piantadosi S, Byar DP and Green SB. The ecological fallacy. American Journal of Epidemiology 1988; 127: 893-904.

[31] Vajdic CM, Er CC, Schaffer A, Dobbins T, Wyld L, Meagher NS, Barrett J, Ward RL, SA Pearson. An audit of cancer of unknown primary notifications: A cautionary tale for population health research using cancer registry data. Cancer Epidemiol 2014; 38: 460-464. 